\title{
CGH microarray studies in idiopathic developmental/ cognitive impairment: association of historical and clinical features and the De Vries Score
}

\author{
Promilla Perattur, Noralane M. Lindor
}

Department of Medical Genetics Mayo Clinic, Rochester

Minnesota, USA

\author{
Corresponding author: \\ Noralane M. Lindor \\ Department of Medical Genetics \\ MA 7-50E \\ Mayo Clinic Rochester \\ Minnesota USA 55902 \\ nlindor@mayo.edu \\ Tel.: + 1-5072662967 \\ Fax: + 1-507 2841067
}

Received: 21 December 2010 Accepted: 23 March 2011

Copyright (C) 2011 by Academy of Sciences and Arts of Bosnia and Herzegovina. E-mail for permission to publish: amabih@anubih.ba
Objective. Studies have confirmed that copy number variations $(\mathrm{CNV})$ in the human genome contribute to the etiology of mental retardation/ development delay/ congenital anomalies. We sought to evaluate the use of a microarray in the context of a clinical genetics practice, to determine if there were any specific clinical findings that predict the discovery of a CNV. Patients and methods. 334 cases with idiopathic mental retardation/impairment/development delay/ disability or a combination of these findings were studied using array comparative genomic hybridization (Signature Chip Version 4). The subjects had previously had a non diagnostic medical genetics evaluation. Clinical findings were collated by a chart review. Each patient was scored according to a previously published clinical checklist by de Vries and colleagues. Results. Of 334 patients, 8 were excluded due to a syndromic diagnosis being established by clinical and/or microarray testing. Out of the remaining 326 patients, 33 (10\%) showed CNVs, of which 5 were maternally inherited, 4 paternally inherited, 11 were de novo, and the origin of 13 remained unknown. The mean de Vries score was greater in the CNV group than in the non CNV group (4.17 and 3.95, respectively). No patient in the CNV group had a score of less than 3, while in the non CNV group, $12 \%$ of patients had scores less than 3. Conclusions. The De Vries clinical score was higher in $\mathrm{CNV}$ cases compared to those with no CNV ( $\mathrm{p}=0.04)$ but this difference is unlikely to be clinically meaningful. Several features reached statistical significance of $\mathrm{p}<0.05$ but we were unable to delineate patterns of features that might increase the yield of positive $\mathrm{CNV}$ results.

Key words: Copy number variation, Developmental delay, Dysmorphism, Birth defects, Prenatal.

\section{Introduction}

Mental retardation/subnormality/impairment/disability/ or developmental delay, with or without other clinical findings, comprises a substantial portion of patients referred to medical geneticists for diagnosis. Around $2 \%$ of the human population has mental retardation, defined by 
the World Health Organization as a "global and noticeable deficiency in the development of motor, cognitive, social, and language functions". The most recent version of the Diagnostic and Statistical Manual of Mental Disorders cites the criteria for diagnosing mental retardation (MR; more commonly now called mental deficiency or mental subnormality) as:

1) Significantly sub-average intellectual functioning: An IQ of approximately 70 or below on an individually administered IQ test. For infants, a clinical judgment for significantly sub-average intellectual functioning, and

2) Concurrent deficits or impairments of onset prior to age 18 years in adaptive functioning in at least two of the following areas: communication, self-care and home living, social skills, use of community resources, self-direction, functional academic skills, work, leisure, health issues, and safety. If onset is after age 18 years, then the diagnosis is dementia (1).

The cause of mental subnormality is identified in only $40-60 \%$ of affected individuals, and a very specific syndrome or genetic diagnosis is found in a few as $20 \%$ $(2,3)$. Recent advances in molecular cytogenetics have increased the diagnostic yield. Historically, traditional karyotyping identified chromosomal aneuploidy in around 14 percent of all cases of severe mental subnormality, with Down syndrome accounting for the majority (4). The ability to detect smaller and smaller copy number variations by use of molecular karyotyping, also called array comparative genomic hybridization $(\mathrm{aCGH})$, has reduced the number of individuals who lack a diagnosis. A review of 13 studies that used bacterial artificial chromosome (BAC) arrays showed abnormalities of copy number in $4-17 \%$ of affected individuals who had apparently normal karyotypes (5). A meta-analysis of 19 studies, most using densely spaced oligonucleotide arrays, had a diagnostic yield of $10 \%$ for convinc- ing abnormalities, but also a $7 \%$ chance of a "false positive", defined as chromosomal variants that were judged to be non causal or were of unknown significance. These facts lead to two important conclusions. First, molecular karyotyping can meaningfully increase the diagnostic yield in individuals with mental subnormality. Second, there is a need for methods to better select candidates for testing as $90 \%$ of those tested have non diagnostic results.

De Vries et al. studied 29 subjects with documented subtelomeric deletions and then developed criteria in the form of a simple check list to describe the pre- and postnatal clinical features that might correlate with abnormal karyotyping (6). The checklist included data on birth history, craniofacial and non facial dysmorphism, congenital malformations and family history. The few studies that have looked at frequencies of CNVs have reported this level of clinical detail about their series, likely because of the lack of availability of this detail.

The aim of the current study was to determine if there were any specific features or combination of clinical findings in our clinical medical genetics practice that was significantly associated with the discovery of $\mathrm{CNV}$ using a clinically available microarray study.

\section{Patients and methods}

This cross-sectional study included the first 334 individuals on whom aCGH was performed in the context of the medical genetics practice at Mayo Clinic, Rochester, Minnesota between 2004 and 2007. All of the individuals had different combinations of cognitive impairment, mental subnormality, development delays, seizures, autism, congenital abnormalities, dysmorphic features, in whom no diagnosis had been achieved prior to their referral to our department. Proper consent had been attained for chart review for research purposes. All patients 
were evaluated by one of the five clinical geneticists in the Department of Medical Genetics and all had a normal G-banded karyotypes of peripheral blood lymphocytes. Each patient was scored according to the proposed criteria of de Vries et al., shown in Table 1 (6). Only patients for whom there was no permission to review their medical record were excluded from this study. No other patients were excluded, although some had some data elements missing, hence the changing denominator for some data elements shown in on-line supplements.

Array CGH was performed on blood from each patient using Signature Chip Version 4 (Signature Genomics Laboratory in Spokane, Washington), which included 622 loci using $1887 \mathrm{BAC}$ clones, to look for subtelomeric or pericentromeric interstitial rearrangements, or alterations in known deletion areas. Once the results for the patients were obtained, the parents of the patients with CNVs were also invited to be tested to determine if the alteration seen was de novo or familial. Out of 33 patients with CNVs, both parents were tested in 20 cases, and in 13, either 1 or neither parents was tested.

A detailed chart review was conducted and more than 200 features were abstracted from each chart regarding maternal health, prenatal, perinatal, and postnatal events, growth, developmental achievements (in- cluding motor, language, speech, psychosocial, cognitive), functional abnormalities, behavioral disorders, autism, attention deficit hyperactivity disorder, congenital anomalies, dysmorphic features, and a detailed family history. All findings were considered of potential importance and were recorded. In addition, each patient was scored strictly according to de Vries Criteria (Table 1) in which each category was given a maximum of 2 points and maximum final score was 10 points.

A detailed family history included known major or minor congenital anomalies, a syndrome diagnosis in a relative, including those caused by chromosomal disorder, history of recurrent miscarriages (two or more in any relative including the mother), diagnosis of chromosomal anomaly in a fetus or child, mental retardation or cognitive impairment in relative, and Sudden Infant Death Syndrome.

Prenatal events included low maternal weight gain during pregnancy (less than 5 pounds), a diagnosis of intrauterine growth restriction (birth weight less than third percentile for gestational age), decreased fetal movements compared to previous pregnancies, and abnormal prenatal ultrasound results. Perinatal data included birth weight, length and head circumference, placental or cord anomalies, immediate newborn problems including respiratory problems, hypo-

Table 1 Checklist for patients, adapted from de Vries et al. (6) A maximum score of 10 points can be achieved. No more than 2 points can be achieved in categories A-E

\begin{tabular}{lc}
\hline Finding & Points Scored \\
\hline A. Family history of mental retardation & 1 \\
Compatible with Mendelian inheritance \\
Incompatible with Mendelian inheritance, including discordant phenotypes within one family \\
$\begin{array}{l}\text { B. Growth retardation of prenatal onset } \\
\text { C. Growth abnormalities of postnatal onset: 1 point each for microcephaly or macrocephaly or short } \\
\text { stature or tall stature }\end{array}$ \\
$\begin{array}{l}\text { D. Two or more facial dysmorphic features, notably hypertelorism, anomalies of the nose or ears } \\
\text { E. Non-facial dysmorphism and congenital abnormalities: maximum of 2 points for hand anomaly (1 } \\
\text { point), heart anomaly (1 point), hypospadias+/- undescended testis (1 point) }\end{array}$ \\
\hline
\end{tabular}


glycemia, seizures, hypotonia, and hyperbilirubinemia.

Post natal growth and development parameters included most recent height, weight and head circumference, and attainment of developmental milestones with regard to motor, speech, language, communication, and cognitive development. Short stature was defined as less than third percentile for age and tall stature as greater than $97^{\text {th }}$ percentile for age. The same percentiles were used to define micro- and macrocephaly. "Later onset" (defined as onset older than 5 months) seizures, hypotonia, or autism spectrum disorders diagnoses were noted. Functional abnormalities of vision and hearing were noted.

Chart abstraction for dysmorphism included abnormal head size, shape, fontanelles, dysmorphic aspects of the forehead (sloping, prominent brow, high or low hairline), unusual formation of the eyebrows (sparse, busy, synophrys), facial asymmetry and midfacial abnormalities; abnormal shape, size or orientation of palpebral fissures; strabismus; epicanthic folds, hyperor hypotelorism, abnormalities of the eyes (aniridia, enophthalmos, abnormalities of the nasolacrimal duct, cornea, retina, optic nerve), abnormal morphology of the ears including pits, tags, or creases; dysmorphism of the nose, lips, philtrum, and abnormalities the mandible, tongue, palate, and dentition.
Non craniofacial abnormalities recorded included defects of the palate, heart, central nervous system (e.g., abnormal MRI), chest wall, nipples, abdominal wall or viscera, internal and external genitalia, spine, long bones and joints, hands, feet, skin and hair.

For the purposes of analysis, all studied individuals were assigned either to the group with no CNV or to the group with CNV. Individual features and bundled features were compared between these two groups and $\mathrm{p}$-values were calculated. A threshold of $\mathrm{p}<0.01$ calculated using Chi square analysis was considered significant, though we also noted those $<0.05$ with interest.

\section{Results}

Out of the total 334 individuals evaluated, $10 \%(n=33)$ had a copy number variation (CNV; details in Supplement 1; note that one individual had two CNVs) that was not known to cause a defined syndrome. Eight of the original 334 patients had a syndromic diagnosis made, sometimes clinically, sometimes with CGH array assistance (e.g., velocardiofacial $22 \mathrm{q}$ deletion). These were excluded from our analysis, so the final dataset included a total of 326 cases of which 293 showed no CNV. Table 2 compares general characteristics between these groups. Of the 33 cases with CNVs, 5 were shown to be maternally inherited, 4 were paternally inher-

Table 2 Description of the individuals included in this study

\begin{tabular}{lll}
\hline Characteristics & CNV found $(\mathrm{n}=33)$ & No CNV detected $(\mathrm{n}=293)$ \\
\hline Average age of males (range) & 10.7 years $(0.8-47)$ & 6.9 years $(0.2-37)$ \\
Average age of females & $8.1(0.5-33)$ & $7.1(0.1-43)$ \\
Average paternal age in years & 30.5 (available on 24) & 31.4 (available on 192) \\
Average maternal age in years & 27.4 (available on 30) & 28.4 (available on 244) \\
$\begin{array}{l}\text { Average parity of mother at time of pregnancy } \\
\text { with proband }\end{array}$ & 1.14 (38\% were primigravid) & 1.2 (36\% were primigravid) \\
Mean number of miscarriages & 0.93 (available information on 29) & 0.62 (available information on 199) \\
\hline
\end{tabular}

$\mathrm{CNV}=$ chromosome copy number variation 
ited, 11 were de novo, and the origin could not be determined in 13 as biparental testing could not be accomplished. All CNVs were included in our analyses, regardless of origin, as inherited CNVs may be pathogenic $(7,8)$ (See Supplement 1 for details).

For all cases, we attempted to generate a score using the criteria of de Vries (Table 1). Due to missing data, especially regarding pregnancy, postnatal growth or family history, not all cases could be scored. We were able to provide a de Vries score for 29 patients with CNVs, and 231 patients without CNVs. A comparison of the two groups is shown in Figure 1.

In our study no patients in the $\mathrm{CNV}$ group had scores lower than 3, while in the $\mathrm{CNV}$ negative group, 28 out of 231 patients

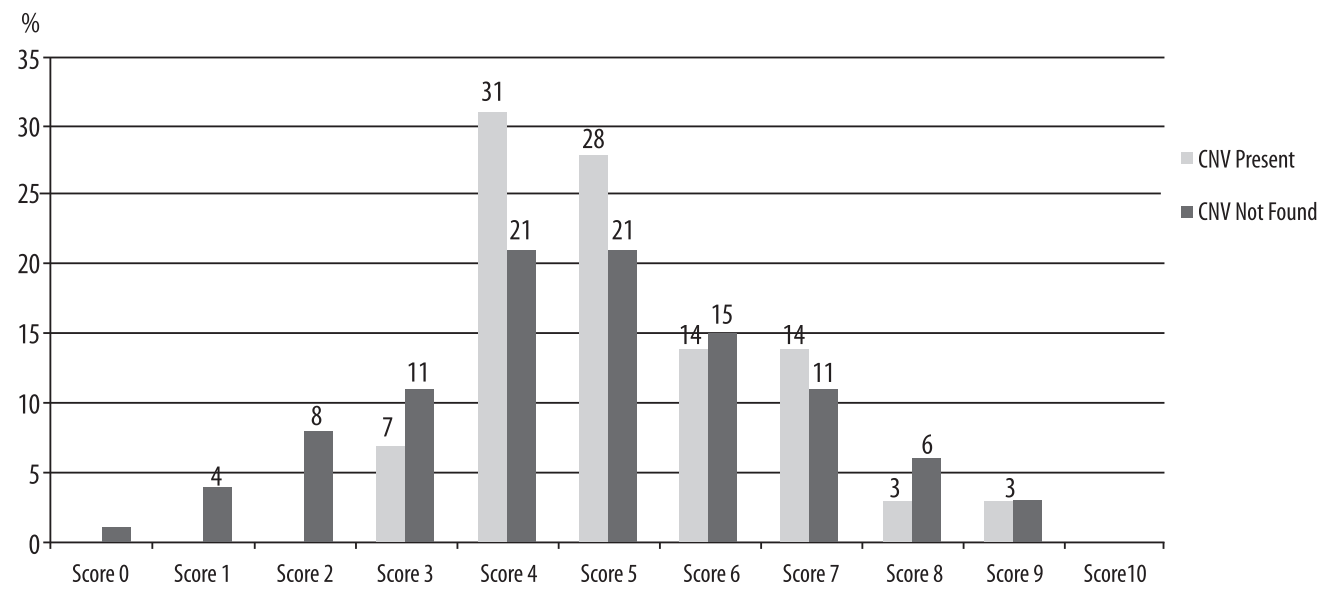

Figure 1 Overall comparison of individuals with CNV detected and those with no detectable CNV using the De Vries scoring system shown in Table 1

Table 3 Analysis of different combinations of scoring in the de Vries table (see Table 1) for cases with sufficient information available to use the de Vries system. P values were all greater than 0.05

\begin{tabular}{|c|c|c|c|c|}
\hline \multirow[t]{2}{*}{ Combinations of scoring in de Vries system } & \multicolumn{2}{|c|}{$\begin{array}{l}\text { CNV } \\
\text { Detected }(n=29)\end{array}$} & \multicolumn{2}{|c|}{$\begin{array}{l}\text { No CNV } \\
\text { Detected }(n=231)\end{array}$} \\
\hline & $\mathrm{n}$ & $\%$ & $\mathrm{n}$ & $\%$ \\
\hline \multicolumn{5}{|l|}{ Prenatal growth retardation } \\
\hline Prenatal growth retardation with 1 or more features & 4 & 14 & 16 & 7 \\
\hline Prenatal growth retardation with 2 or more features & 3 & 10 & 15 & 6 \\
\hline \multicolumn{5}{|l|}{ Postnatal growth abnormality } \\
\hline Postnatal growth abnormalities with 1 or more features & 16 & 55 & 101 & 44 \\
\hline Postnatal growth abnormalities with 2 or more features & 4 & 14 & 45 & 20 \\
\hline \multicolumn{5}{|l|}{ Family history positive } \\
\hline Family history with 1 or more features & 19 & 66 & 138 & 60 \\
\hline Family history with 2 or more features & 7 & 24 & 37 & 16 \\
\hline \multicolumn{5}{|l|}{ Facial dysmorphism } \\
\hline Facial dysmorphism with 1 or more features & 24 & 83 & 205 & 89 \\
\hline Facial dysmorphism with 2 or more features & 20 & 69 & 186 & 81 \\
\hline \multicolumn{5}{|l|}{ Nonfacial dysmorphism } \\
\hline Nonfacial dysmorphism with 1 or more features & 28 & 97 & 198 & 86 \\
\hline Nonfacial dysmorphism with 2 or more features & 23 & 79 & 165 & 71 \\
\hline
\end{tabular}

$\mathrm{CNV}=$ copy number variation 
(12\%) had scores of 2 or lower. The average de Vries score in the CNV group was 4.17 and in the CNV negative group was 3.95 $(\mathrm{p}=0.04)$. In Table 3, different combinations of findings were compared between the $\mathrm{CNV}$ and non $\mathrm{CNV}$ groups but none seemed to select for $\mathrm{CNV}$ cases efficiently.

The presence or absence of over 200 specific individual findings beyond the deVries criteria were also evaluated and compared between individuals with CNVs and those with no CNV. A statistically significant difference was seen at the $\mathrm{p}<0.05$ level (but no $\mathrm{p}$ values were less than 0.01 ) in the frequency of specific features was noted between the $\mathrm{CNV}$-positive and the CNV-negative groups in 3 categories (full details in Supplements 2-6):

1) Developmental delay within first 5 months in the CNV group was reported in $21 \%$ and in the non $\mathrm{CNV}$ group in $6 \%$ $(\mathrm{p}=0.020)$ and

2) Congenital hypotonia was observed in $28 \%$ of CNV cases while only in $12 \%$ of non CNV cases $(\mathrm{p}=0.0496)$.

3) Macrocephaly, excluding relative macrocephaly, was present in $17 \%$ of $\mathrm{CNV}$ cases versus $5 \%$ in non-CNV cases $(\mathrm{p}=0.026)$.

Trends favoring a higher frequency in those with CNVs were observed for intrauterine growth retardation $(\mathrm{p}=0.051)$, two or more miscarriages in the mother $(\mathrm{p}=0.076)$, and a history of decreased fetal movements as felt by the mother in this pregnancy as compared to previous ones $(\mathrm{p}=0.067)$.

\section{Discussion}

In this study we sought to determine if there were any specific clinical features that individually or in combination would provide increased yield of CNVs when studying individuals with aCGH. Three hundred and thirty four chromosomally normal individuals having diagnostic evaluations in the Department of Medical Genetics for developmental delay, with or without other anomalies, were included in this retrospective chart review. The major strength and novelty of this study was access to very detailed and quite complete clinical information, allowing comparison between those with CNVs to those with no findings. We also applied the checklist proposed by de Vries to this cohort to determine its utility (6). Our results showed trends toward higher scores using the de Vries check list, and several individual features were identified that may merit further clinical studies of this type. Overall, however, no finding was found at a frequency that was sufficiently different between the CNV group and the group without CNVs to inform changes in current clinical practice.

This finding is not surprising and several explanations likely contribute to this finding. The first generation clinical microarray was targeted toward areas of known clinical significance and the coverage in other parts of the genome was poor. The newer oligoarray studies yield modestly increased numbers of CNVs in similar populations. Undoubtedly there could be individuals in our study that had CNVs not detected on this first generation microarray (5, 9-12). Secondly, there may not be a phenotype resulting from CNVs that is so characteristic as to distinguish it from non genetic causes of mental subnormality, with or without birth defects. Also, single gene disorders can cause mental subnormality, dysmorphism and birth defects, and none of these will be detected by any type of CGH array.

In our analyses, we chose to include all $\mathrm{CNVs}$ regardless of whether they were familial or de novo, rejecting the assertion that a familial CNV will be non causal. We have observed a number of families in which the carrier parent was functioning adequately but appeared to share phenotypic features with the proband. In addition, further genetic change in the size of a familial CNV 
across meiosis has also been reported $(7,8)$. As some familial CNVs may truly be without consequences, we may have diluted our "affected" group by making this choice.

\section{Conclusion}

De Vries clinical score was higher in CNV cases compared to those with no CNV $(\mathrm{p}=0.04)$ but this difference is unlikely to be clinically meaningful. Several individual features reached statistical significance at the $\mathrm{p}<0.05$ threshold but we were unable to delineate patterns of features that might increase the yield of positive $\mathrm{CNV}$ results. What we can do as clinicians to increase our ability to increase the diagnostic yield of tests we order? Can we make a more informed selection of candidates for microarray testing? It will be of interest to see this type of clinical correlative analysis repeated on the newer platforms used for molecular karyotyping. Only by detailed reflection on our outcomes will we know if we could be practicing in a more efficient manner.

Authors' contributions: Conception and design: PP and NML; Acquisition, analysis and interpretation of data: PP and NML; Drafting the article PP and NML; Revising it critically for important intellectual content: PP and NML.

Conflict of interest: The authors declare that they have no conflict of interest. This study was not sponsored by any external organization.

\section{References}

1. American Psychiatric Association. Diagnostic and Statistical Manual of Mental Disorders. Washington, DC: American Psychiatric Publishing, Inc.; 2000.

2. Curry CJ, Stevenson RE, Aughton D, Byrne J, Carey JC, Cassidy S, et al. Evaluation of mental retar- dation: recommendations of a Consensus Conference: American College of Medical Genetics. Am J Med Genet. 1997;72(4):468-77.

3. Hunter AG. Outcome of the routine assessment of patients with mental retardation in a genetics clinic. Am J Med Genet. 2000;90(1):60-8.

4. Opitz JM, Kaveggia EG, Durkin-Stamm MV and Pendleton E. Diagnostic/genetic studies in severe mental retardation. Birth Defects Orig Artic Ser. 1978;14(6B):1-38.

5. Shao L, Shaw CA, Lu XY, Sahoo T, Bacino CA, Lalani SR, et al. Identification of chromosome abnormalities in subtelomeric regions by microarray analysis: a study of 5,380 cases. Am J Med Genet A. 2008;146A(17):2242-51.

6. de Vries BB, White SM, Knight SJ, Regan R, Homfray T, Young ID, et al. Clinical studies on submicroscopic subtelomeric rearrangements: a checklist. J Med Genet. 2001;38(3):145-50.

7. South ST, Rope AF, Lamb AN, Aston E, Glaus N, Whitby $\mathrm{H}$, et al. Expansion in size of a terminal deletion: a paradigm shift for parental follow-up studies. J Med Genet. 2008;45(6):391-5.

8. Merritt JL and Lindor NM. Further clinical description of duplication of Williams-Beuren region presenting with congenital glaucoma and brachycephaly. Am J Med Genet A. 2008;146A(8):1055-8.

9. Shevell MI, Bejjani BA, Srour M, Rorem EA, Hall $\mathrm{N}$ and Shaffer LG. Array comparative genomic hybridization in global developmental delay. Am J Med Genet B Neuropsychiatr Genet. 2008;147B(7):1101-8.

10. Hoyer J, Dreweke A, Becker C, Göhring I, Thiel CT, Peippo MM, et al. Molecular karyotyping in patients with mental retardation using $100 \mathrm{~K}$ single-nucleotide polymorphism arrays. J Med Genet. 2007;44(10):629-36.

11. Sagoo GS, Butterworth AS, Sanderson S, ShawSmith C, Higgins JP and Burton H. Array CGH in patients with learning disability (mental retardation) and congenital anomalies: updated systematic review and meta-analysis of 19 studies and 13,926 subjects. Genet Med. 2009;11(3):139-46.

12. Bernardini L, Alesi V, Loddo S, Novelli A, Bottillo I, Battaglia A, et al. High-resolution SNP arrays in mental retardation diagnostics: how much do we gain? Eur J Hum Genet. 2010;18(2):178-85. 
Acta Medica Academica 2011;40:17-26

\begin{tabular}{|c|c|c|c|c|}
\hline Origin of CNV & Deletion & BAC clones & Duplication & BAC clones \\
\hline De novo & $19 p 13.3$ & 3 & - & - \\
\hline De novo & $22 q 13.33$ & 1 & - & - \\
\hline De novo & $1 q 44$ & 23 & - & - \\
\hline De novo & $20 q 11.23$ & 3 & - & - \\
\hline De novo & $5 q 35.2$ & $\sim 3 \mathrm{MB}$ & - & - \\
\hline De novo & $1 q 41 q 42.12$ & 9 & - & - \\
\hline De novo & $13 q 34$ & 26 & $18 q 23$ & 2 \\
\hline Mat & $6 \mathrm{p} 21.1$ & 2 & - & - \\
\hline Mat & $11 \mathrm{p} 15.4$ & 3 & - & - \\
\hline pat & $11 \mathrm{p} 12$ & 1 & - & - \\
\hline pat & $6 \mathrm{p} 25.1$ & 3 & - & - \\
\hline Unknown & $2 q 13$ & 1 & - & - \\
\hline Unknown & $17 p 13.2$ & 2 & - & - \\
\hline Unknown & $6 q 16.3$ & 9 & - & - \\
\hline Unknown & $6 q 27$ & 3 & - & - \\
\hline Unknown & $2 q 13$ & 1 & - & - \\
\hline Unknown & $6 q 27$ & 14 & - & - \\
\hline Unknown & $2 q 13$ & 1 & - & - \\
\hline De novo & - & - & $1 q 44$ & 24 \\
\hline De novo & - & - & $2 \mathrm{p} 25.3$ & $\mathrm{RP11-1N7} \rightarrow$ RP11-97B21 \\
\hline De novo & - & - & $11 \mathrm{p} 12 \mathrm{p} 11.12$ & 25 \\
\hline De novo & - & - & $18 q 23$ & 2 \\
\hline Mat & - & - & $17 p 13.3$ & 2 \\
\hline Mat & - & - & Xp22.31 & 3 \\
\hline Mat & - & - & $7 q 11.23$ & CTD--2069P19 $\rightarrow$ RP11-97B21 \\
\hline pat & - & - & Yp11.32 & 3 \\
\hline pat & - & - & $8 q 24.3$ & 2 \\
\hline Unknown & - & - & $10 p 15.3$ & 3 \\
\hline Unknown & - & - & $15 q 26.3$ & 3 \\
\hline Unknown & - & - & $1 p 36.3$ & $\begin{array}{l}\text { Duplication of } 3 \mathrm{BAC} \text { and } \\
\text { triplication of } 3 \mathrm{BAC}\end{array}$ \\
\hline Unknown & - & - & $20 \mathrm{p} 12.2$ & 3 \\
\hline Unknown & - & - & $11 \mathrm{p} 13$ & $\begin{array}{l}\text { RP11-293B1, RP11-26B16 } \\
\text { RP11-133E13 }\end{array}$ \\
\hline Unknown & - & - & $7 q 11.23$ & 6 \\
\hline Unknown & - & - & $8 p 23.3$ & RP11-388B22 \\
\hline
\end{tabular}

Supplement 1 Summary of the CVNs included in this study 


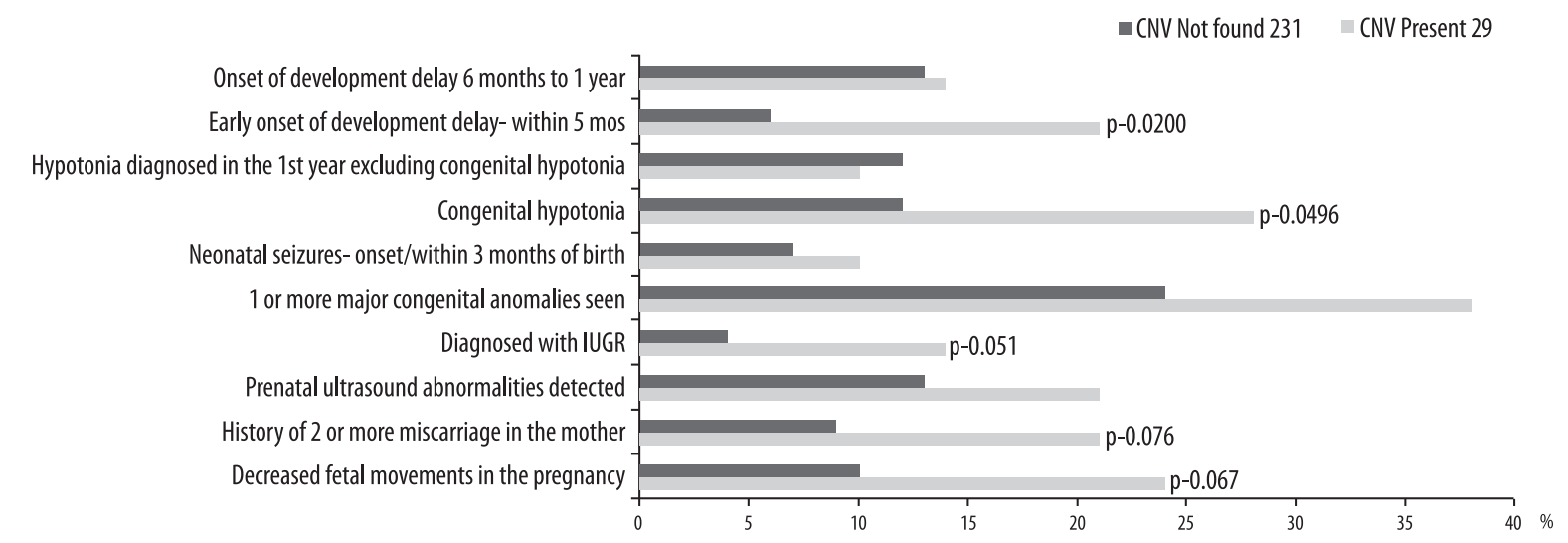

Supplement 2 Comparisons of specific features for the history between 33 individuals with CNV detected and 293 with no CNV detected. CNV=copy number variation

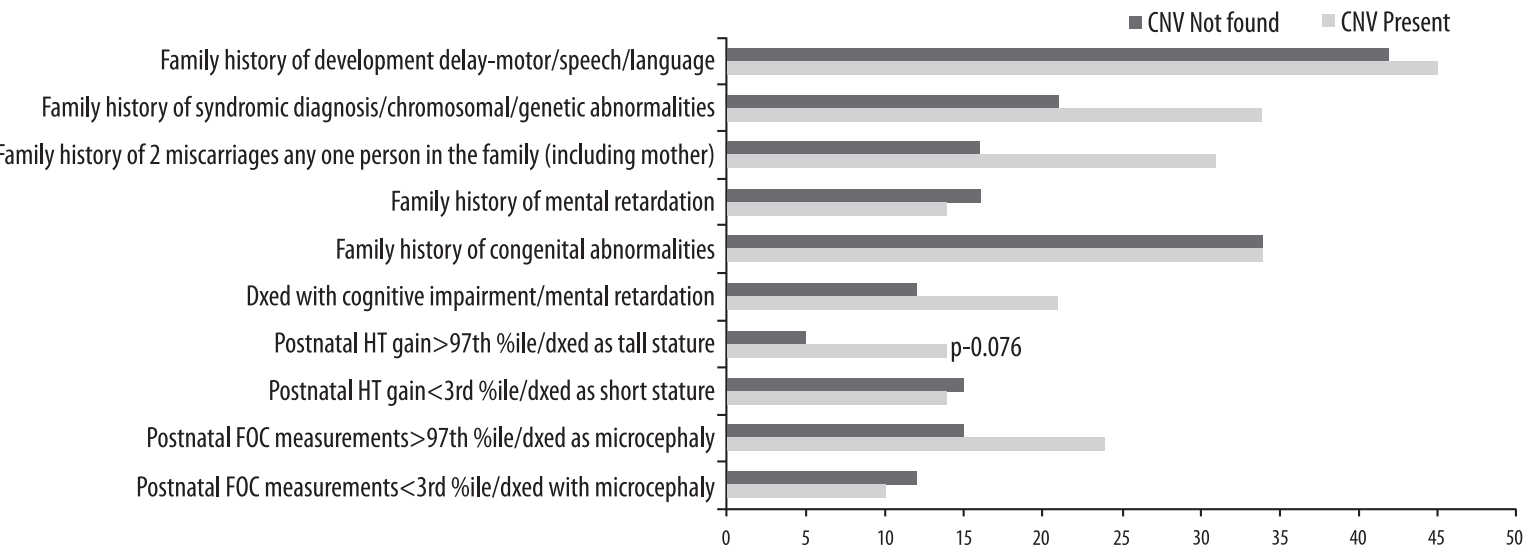

Supplement 3 Comparisons of additional historical features between 33 individuals with CNV detected and 293 with no CNV detected. Using a p value of less than 0.01 , none were statistically significantly different. $\mathrm{CNV}=$ copy number variation

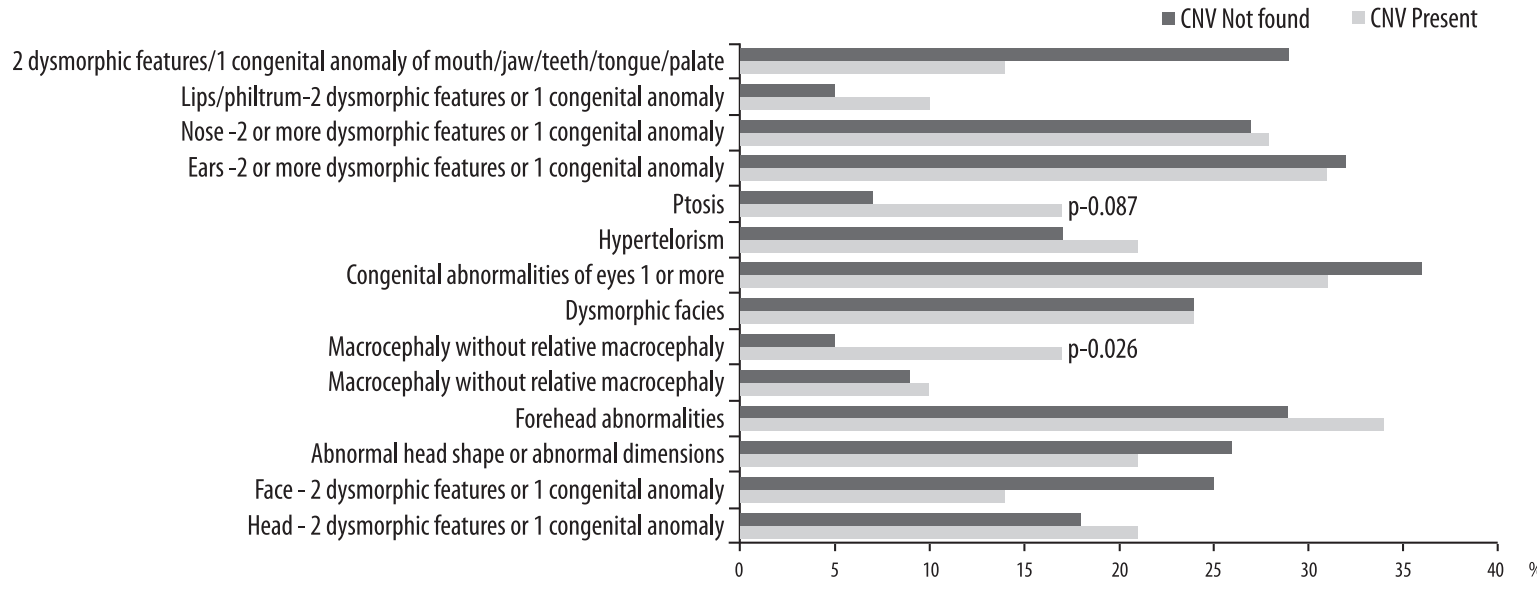

Supplement 4 Comparison of craniofacial features between 33 individuals with CNV detected and 293 with no CNV detected. Using a p value of less than 0.01 , none were statistically significantly different. CNV=copy number variation 
Skeletal abnormalities including joints - 2 dysmorphic features $/ 1$ congenital anomaly 1 dysmorphic feature of feet Hand - 1 dysmorphic feature 1 skin abnormalities of any type Hair abnormalities - minimum 1 dysmorphic feature 1 congemital genital/lower urinary system abnormalities Gastrointestinal anomaly of any typ Congenital central nervous system abnormalities found in MRI/CT scan Al anomaly/abnormalities of CNS including congenital hypotoni and congenital microcephaly/macrocephaly Congenital heart disease

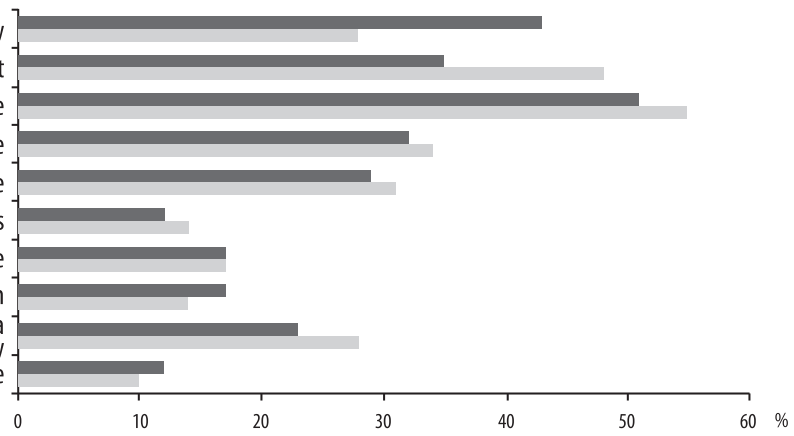

Supplement 5 Comparison of noncraniofacial features between 33 individuals with CNV detected and 293 with no CNV detected. $C N V=$ copy number variation. Using a $p$ value of less than 0.01 , none were statistically significantly different.

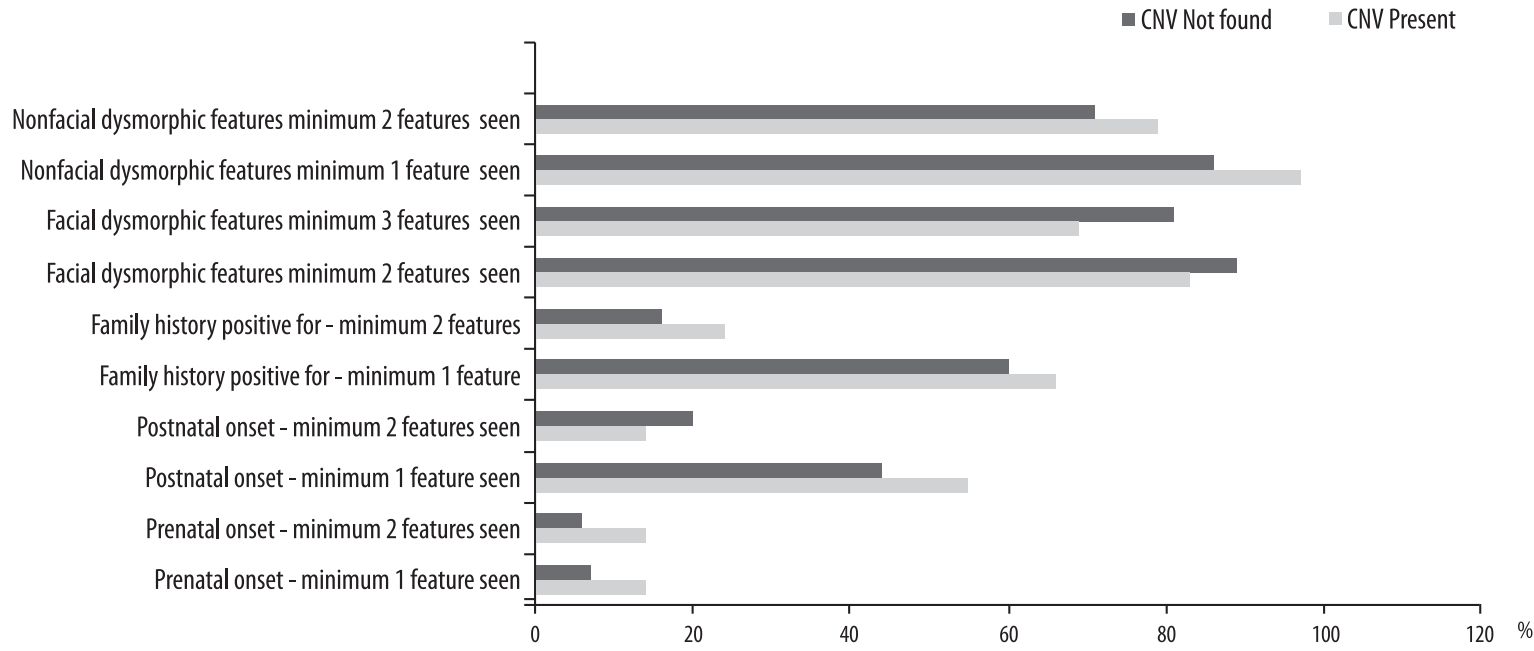

Supplement 6 Comparison of different combinations of findings between the CNV and non CNV groups. Using a $p$ value of less than 0.01 , none were statistically significantly different. 